\title{
Fatigue craze initiation in polycarbonate: study by transmission electron microscopy
}

\author{
Hristo A. Hristov, Albert F. Yee* and David W. Gidley $\dagger$ \\ Department of Materials Science and Engineering and †Department of Physics, University \\ of Michigan, Ann Arbor, MI 48109, USA \\ (Received 22 October 1993; revised 26 January 1994)
}

\begin{abstract}
Fatigue craze initiation in bulk, amorphous polycarbonate $(\mathrm{PC})$ is investigated by means of transmission electron microscopy. The results agree very well with small angle X-ray scattering measurements of the same set of samples, and confirm that void generation is the main characteristic of the initiation process. The evolution of the initial void-like structures or 'protocrazes' with dimensions of $\sim 50 \mathrm{~nm}$ into crazes with dimensions of several micrometres is presented in considerable detail. It is found that some similarities exist between crazes induced by cyclic fatigue at room temperature and crazes produced in monotonic loading at temperatures close to the glass transition. The similarities suggest that disentanglement of polymer chains plays an important role in the fatigue craze initiation process in bulk, amorphous PC near room temperature.
\end{abstract}

(Keywords: fatigue; craze initiation; polycarbonate)

\section{INTRODUCTION}

The importance of crazing in the structural integrity of polymeric materials under load was recognized more than 20 years ago, which prompted the beginning of systematic investigations of structure of crazes in polymers by, among other techniques, transmission electron microscopy (TEM) ${ }^{1-4}$. The majority of the experimental observations has been devoted to determining the morphological parameters of large 'mature' crazes introduced by creep and monotonic loading in various homopolymers, copolymers, blends and composites. These studies have established (most of the existing information is summarized in several review articles ${ }^{5-11}$ ) that a typical craze formed under uniaxial deformation is characterized by a network of almost cylindrical fibrils, oriented nearly perpendicular to the craze/bulk interface and parallel to the external stress. The fibrils are composed of highly drawn (oriented) polymeric material, and are often interconnected by 'cross-ties'. The craze morphology and fibril diameters may change depending on the type of the polymer (e.g. high or low entanglement density polymer, molecular weight, crosslinks), and external variables such as temperature, rate of deformation, crazing agents, specimen thickness and a few other factors $5,6,12-16$. Craze initiation in thin polystyrene (PS) films $\mathrm{s}^{17}$ and in thicker PS materials ${ }^{15.16}$ has been studied under monotonic loading conditions. It has been found that the formation of localized surface deformation zones or precraze 'furrows' leads to the generation of small voids, which coalesce and grow into large, mature crazes.

Despite the relative abundance of microscopic data, TEM investigations of craze and precraze structures produced by cyclic fatigue have not been reported to date. To the best of our knowledge, microscopic models

* To whom correspondence should be addressed describing fatigue craze initiation have not been developed either (reviews of the existing models of craze initiation under constant load can be found in references 9 and 16). It seems that optical microscopy (conventional and interference optical microscopy) is the most frequently used method for studying the gross structure and the dynamics of fatigue induced cracks and crazes $^{18-21}$. Fatigue fracture surfaces of many polymers have been studied by optical microscopy and scanning electron microscopy (SEM) ${ }^{18-22}$. SEM has been used to elucidate the morphology of a single craze propagating in front of a fatigue induced crack in bulk poly(vinyl chloride) (PVC) ${ }^{19,22}$. It has been found that, similarly to the crazes formed at constant load, the craze material formed in fatigue consists of fibrils spanning the craze zone; however the fibril diameter $(\sim 200 \mathrm{~nm})$ appears to be an order of magnitude larger than that characteristic for the constant load counterpart.

Fatigue craze initiation in semicrystalline poly(oxymethylene) (POM) has been studied by small angle X-ray scattering (SAXS) ${ }^{23}$. The SAXS method has also been used to follow in situ the changes of the craze structure in PS under conditions of cyclic bending at high amplitudes ${ }^{24}$, and in several rubber modified thermoplastics during tensile impact ${ }^{25}$. It has been established that the SAXS method has a number of advantages ${ }^{26}$ when used to characterize crazes in bulk specimens, but the method has its limitations: first, the information is averaged over at least several structural agglomerations, which introduces some ambiguity in the data interpretation; and second, by nature a SAXS measurement lacks the local detail inherent to electron microscopy. In addition the maximum size that can be measured in the majority of the SAXS instruments is limited to $200-300 \mathrm{~nm}$, thus the gap between $200-300 \mathrm{~nm}$ and $1 \mu \mathrm{m}$ (the resolution limit of the conventional optical microscopy) can be successfully 
covered by the electron microscopy methods. It should be noted that a combination of several structural methods is frequently employed to study craze structures in polymers (TEM and SAXS can be regarded as complementary in this respect) ${ }^{10,11,26}$.

In a preceding paper, we have reported SAXS investigations of fatigue craze initiation in bulk amorphous polycarbonate $(\mathrm{PC})^{27}$. The results indicate that the fatigue process leads to the development of oblate void-like formations with dimensions $\sim 60 \mathrm{~nm}$ in the direction parallel to the fatigue stress, and $150-200 \mathrm{~nm}$ in the transverse direction. With increasing numbers of fatigue cycles at constant stress amplitude, these 'protocrazes' ultimately transform into mature crazes, although the details of the transformation process are not clear. In the present work, we report results from a TEM study of the same set of samples ${ }^{27}$. The main goal is to provide direct structural information by an experimental method other than SAXS, and if possible to eliminate any ambiguities with regard to the structural elements characterizing fatigue craze initiation in bulk, amorphous PC. The second objective of the study is to obtain detailed information describing the transformation of the 'protocrazes' into visible crazes.

A fundamental problem of any TEM investigation of bulk samples is the unavoidable microtoming and possible damage resulting from the cutting; however, the shapes of relatively simple structural formations, such as voids, should be easy to recognize. One of the objections of microtoming bulk specimens containing crazes produced under constant or monotonic loads is the possible change of the craze morphology (relaxation of the craze structure and fibril coalescence ${ }^{2,4-6,24}$ ) when the stress is reduced to zero. Taking into account that the stress is reduced to zero thousands of times in the course of the cyclic fatigue (see the Experimental section) such an objection does not exist for crazes generated in fatigued samples. An important specific regarding PC, is the fact that it crazes under cyclic load ${ }^{22}$, but yields under monotonic loading at ordinary strain rates, apparently due to its high entanglement density, and creates deformation zones ${ }^{28}$ at room temperature. As a result, the probability of introducing new crazes during the post-fatigue specimen handling (which may involve low level stress of relatively simple history) is very low. It may be argued that fatigued PC is much more susceptible to crazing. While this is a definite possibility it can be avoided or at least distinguished by varying the angle of cutting with respect to the original loading direction.

\section{EXPERIMENTAL}

The samples investigated in the present work were prepared from compression moulded PC resin supplied by the General Electric Co. The moulding conditions have been described previously ${ }^{29,30}$. The moulded plaques were examined optically and it was found that they are free of gas bubbles and there is no preferred orientation (zero birefringence). The smooth dumb-bellshaped specimens with thickness $3 \mathrm{~mm}$ were machined at room temperature and the edges were polished manually. To remove the residual stresses, induced by the cutting procedure the test samples were annealed at $160^{\circ} \mathrm{C}$ (after vacuum drying at $100^{\circ} \mathrm{C}$ for $12 \mathrm{~h}$ ) for $40 \mathrm{~min}$ and cooled rapidly to room temperature. Prior to the cyclic loading the specimens were aged for $24 \mathrm{~h}$ at $130^{\circ} \mathrm{C}$.
The fatigue procedures were performed on a computercontrolled servohydraulic machine (Instron, model 1332), at room temperature, under a constant stress amplitude of $30 \mathrm{MPa}\left(\sigma_{\min } / \sigma_{\max }=0.05\right.$; sinusoidal) and frequency of $5 \mathrm{~Hz}$. More details on the materials, thermal treatment and instrumentation can be found in references 27,29 and 30 .

Since at this load amplitude the samples fracture after $9000-10000$ cycles, a set of specimens was prepared by stopping the fatigue process after $1000,3000,5000$ and 7000 cycles (designated as samples $1 \mathrm{~K}, 3 \mathrm{~K}, 5 \mathrm{~K}$ and $7 \mathrm{~K}$, respectively). Optical microscopy investigations revealed that visible crazes can be found in specimens $5 \mathrm{~K}$ and $7 \mathrm{~K}$, while samples $1 \mathrm{~K}$ and $3 \mathrm{~K}$ appear to be homogeneous, SAXS measurements showed that in some portions of the fatigued specimens one can detect the presence of oblate void-like formations, which were termed 'protocrazes' ${ }^{27}$. Those regions of the samples $1 \mathrm{~K}, 3 \mathrm{~K}$, $5 \mathrm{~K}$ and $7 \mathrm{~K}$ exhibiting $\mathrm{X}$-ray diffraction characteristic for the protocrazes and some of the crazed parts of specimens $5 \mathrm{~K}$ and $7 \mathrm{~K}$ were cut by diamond saw as shown schematically in Figure 1. Some of the sections were stained in $\mathrm{OsO}_{4}$ vapour for $60 \mathrm{~h}$ before microtoming. The microtomy was effected with a diamond knife in a 'Reichert-Jung' ultramicrotome, at room temperature. The microtomed slices, with thicknesses from $40 \mathrm{~nm}$ to $\sim 1 \mu \mathrm{m}$, were lifted off the water trough on copper grids (mesh 200) and dried at room temperature. Different sections of the samples were microtomed along four directions, chosen in such a manner that the stress direction is always in the plane of the thin slices (Figure 1). By choosing the coordinate system as in Figure 1 the microtomy was executed as follows:

- Cut $\mathrm{C} 1$ - the direction of the microtome movement is parallel to the $\mathrm{Y}$ axis (the stress direction); the microtome blade is parallel to the XY plane, and its edge is parallel to the $\mathrm{X}$ axis.

- Cut C2 - the direction of the microtome movement is perpendicular to the stress direction; the microtome

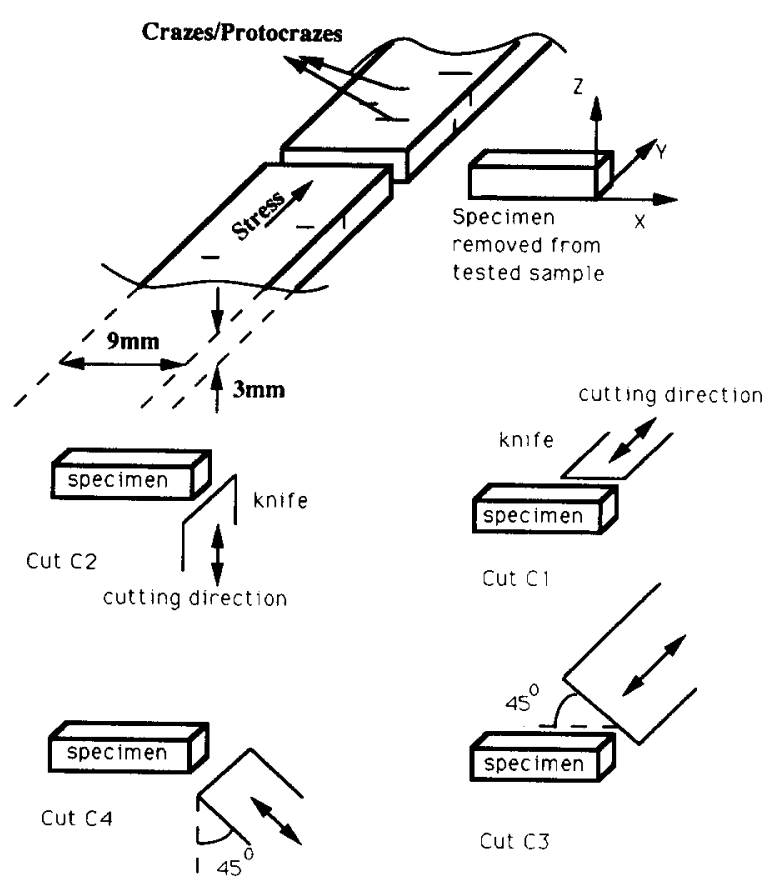

Figure 1 Microtoming geometry 
blade is perpendicular to the $\mathrm{XY}$ plane, and its edge is parallel to the $\mathrm{Y}$ axis.

- Cut $\mathrm{C} 3$ - the direction of the microtome movement is parallel to the stress direction; the microtome blade is at angle $\sim 45^{\circ}$ to the $X Y$ plane, and the projection of its edge on $\mathrm{XY}$ plane is parallel to the $\mathrm{X}$ axis.

- Cut C4 - the direction of the microtome movement is perpendicular to the stress direction; the microtome blade is at angle $\sim 45^{\circ}$ to the XY plane and its edge is parallel to the $\mathrm{Y}$ axis.

Most of the specimens were examined in a JEOL 4000 electron microscope, equipped with a 'Gatan' image intensifier, at an accelerating voltage of $400 \mathrm{kV}$. Since PC, like most polymers, is electron beam sensitive, a few specimens were examined at a lower accelerating voltage $(120 \mathrm{kV})$ using a Philips 420 electron microscope. In the course of the measurements we found that, if the microtomed sections are thinner than $\sim 300 \mathrm{~nm}$ then it is possible to obtain clear micrographs, with minimal beam damage, by using either instrument. For thicker sections the higher accelerating voltage gives much better results.

The results from the fatigued samples were compared to results obtained from unfatigued PC specimens, and fatigued PC samples containing 10\% PS (PC-PS samples). Since the PC-PS are immiscible the PS forms spherical particles with average size $\sim 100 \mathrm{~nm}$ in the PC matrix. (A graft copolymer of PC- $g$-PS is used to compatibilize the two phases. Results from the fatigue of this system will be reported in a subsequent paper). In both cases the specimens were microtomed as in $\mathrm{C} 1$. The morphology of the fatigue induced crazes was compared, also, to the morphologies of crazes produced by high temperature uniaxial deformation of solution cast PC film in a manner similar to that described by Kramer and Berger ${ }^{10}$. The $\sim 0.5 \mu$ m thick PC film was bonded to a copper grid and deformed at a rate of $\sim 1 \%$ $\min ^{-1}$ at $125^{\circ} \mathrm{C}$, until the material crazed extensively. Representative grid squares were then removed for observation, with no further preparation.

\section{RESULTS AND DISCUSSION}

The fatigue craze initiation process was documented starting with the smallest structural features or 'protocrazes'. The increase of the protocraze dimensions was followed step by step until the protocrazes transformed into crazes with dimensions in the micrometre range.

\section{Structural characteristics of the craze initiation (samples $1 K, 3 K, 5 K$ and $7 K$ )}

Figure 2 shows a collection of the smallest isolated void-like formations found in the fatigued materials. Despite some damage from the knife, it is clear that their general shape is oblate, with sizes varying from 40 to $80 \mathrm{~nm}$, and an aspect ratio of $\sim 2$. This kind of voids or protocrazes was observed in thin sections $(40-50 \mathrm{~nm}$; Figures $2 a-c$ ) obtained from appropriate regions of all of the fatigued specimens. No such features were found in slices from the unfatigued control samples, despite exhaustive searches. It is known that the microtome can produce holes in very thin slices, however such holes are always in the root of a knife mark; no knife marks are visible in Figure 2. As a rule the microtome produces rows of holes rather than a single isolated one, and the microtome induced holes are elongated in the direction of the knife movement. In Figures $2 a, b$ and $d$ the long dimension is perpendicular to the knife movement.

It was found also that the protocrazes are situated predominantly within a layer $0-500 \mu \mathrm{m}$ below the surfaces of the samples. Investigations of sections from the central part of the fatigued specimens revealed a homogeneous amorphous structure, visually identical to that observed in the non-fatigued material. In thicker sections $(\sim 100 \mathrm{~nm})$ the void image is blurred as shown in Figure $2 d$, and in slices thicker than $\sim 200 \mathrm{~nm}$ the initial voids are not observable. The latter results probably from a combination of factors, namely in thick sections the images are poorly resolved and in addition the smallest voids may be closed (at least partially) by the stress field of the surrounding material. (In thin slices

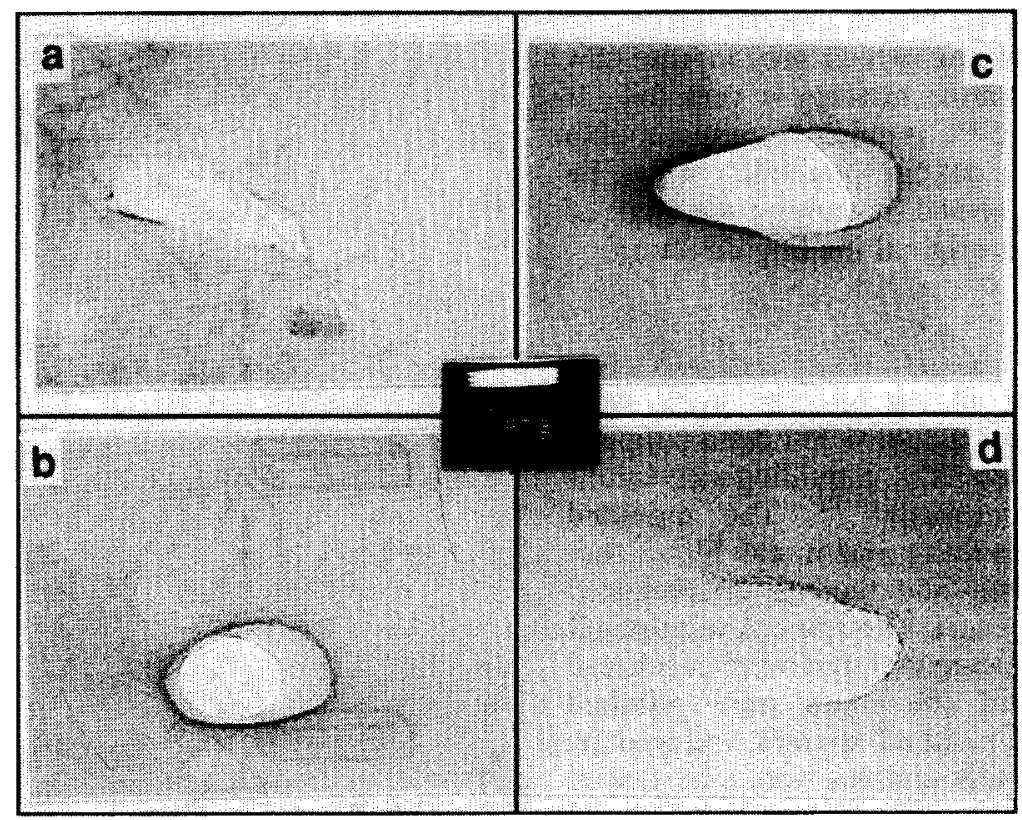

Figure 2 Isolated voids in the fatigued specimens: (a) sample $1 \mathrm{~K}$, cut $\mathrm{C1}$; (b) sample $3 \mathrm{~K}$, cut $\mathrm{C1}$; (c) sample $3 \mathrm{~K}$, cut 4 ; (d) sample $5 \mathrm{~K}$, cut $\mathrm{C} 1$. Fatigue stress axis vertical 


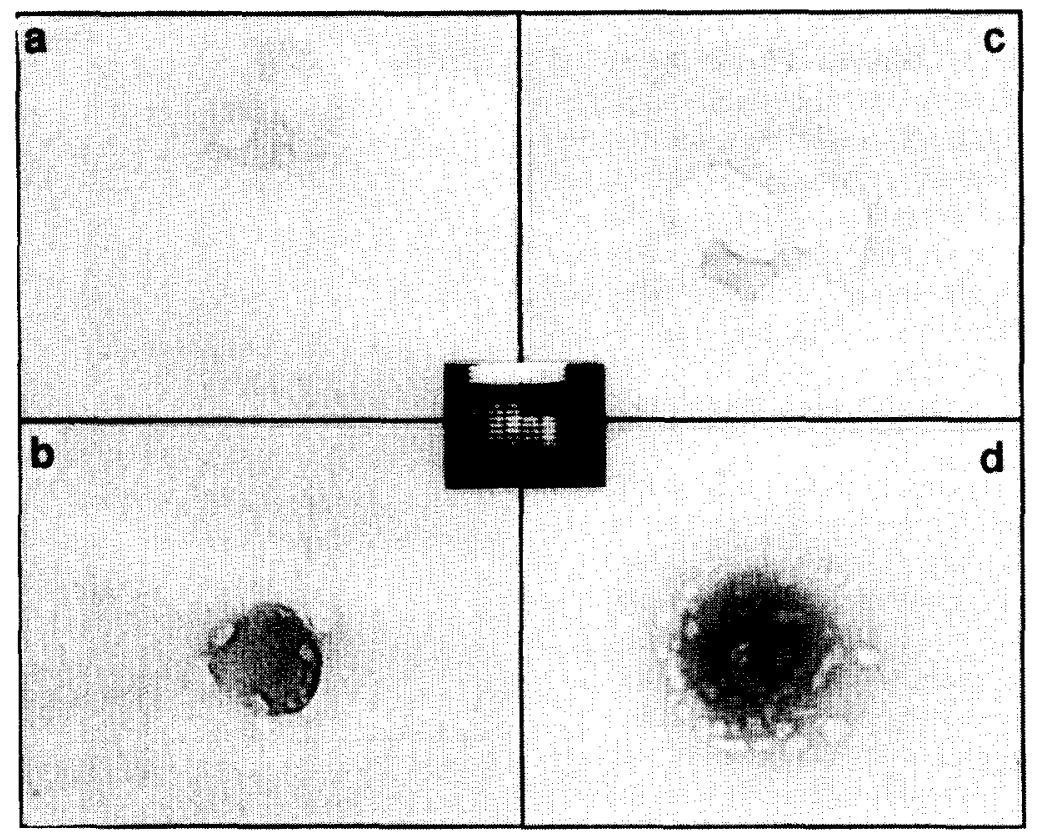

Figure 3 Multiple voiding: (a) sample $3 \mathrm{~K}$, cut $\mathrm{Cl}$; (b) sample $5 \mathrm{~K}$ (voiding caused by a dirt particle), cut $\mathrm{Cl}$; (c) sample $7 \mathrm{~K}$, cut $\mathrm{C} 2$; (d) sample PC-PS (voiding within and around a PS particle), cut $\mathrm{C} 1$. Fatigue stress axis vertical

the situation is exactly the opposite: the resolution is enhanced while the stress is relaxed to zero.)

Our observations revealed also that in some cases the material contains groups of closely clustered irregular voids as shown in Figures $3 a$ and $c$. The presence of multiple voids is a common event if the specimen contains inclusions (dirt) (Figure $3 b$ ) or PS particles (Figure $3 d$, the darkening is a result of moderate beam damage). From Figure 3 it is clear that when the conditions are appropriate for multiple voiding, the dimensions of the smallest void can be $<10 \mathrm{~nm}$, i.e. several times smaller than the size of the smallest isolated void. The observations presented in Figures 2 and 3 suggest that isolated small voids are unstable, and that the critical void size in fatigued $\mathrm{PC}$ is in the range $50-60 \mathrm{~nm}$. Figures $3 b$ and $d$ demonstrate that extraneous particles (which act as stress concentrators) with diameters $50-100 \mathrm{~nm}$ serve as effective craze nucleation sites. Figures $3 b$ and $d$ indicate also that the void initiation, in the presence of stress concentrating particles with spherical symmetry, is independent of the direction of the fatigue stress.

Figure 4 shows the next stage of the protocraze development, when the small dimension reaches 50-100 $\mathrm{nm}$, and the lateral one $150-250 \mathrm{~nm}$. The comparison between Figures 2 and 4 suggests that the protocrazes grow predominantly by expansion in the lateral direction (perpendicular to the fatigue stress axis - Figures $4 a$ and b), or by the coalescence of several voids (Figure $4 c$ ). Careful examination of the formation in Figure $4 \mathrm{c}$ reveals that the protocraze is composed of at least three voids elongated perpendicularly to the fatigue stress. The size of the polymer ligaments between the voids in Figure $4 \mathrm{c}$ ranges from 20 to $50 \mathrm{~nm}$. There is no evidence that the ligaments are created by fusion of finer fibrils, a phenomenon known to take place during the fatigue crack/craze propagation phase ${ }^{22}$. According to Doll ${ }^{22}$ the fusion of fibrils is a consequence of the very high stress intensity (concentration) and the resulting high energy

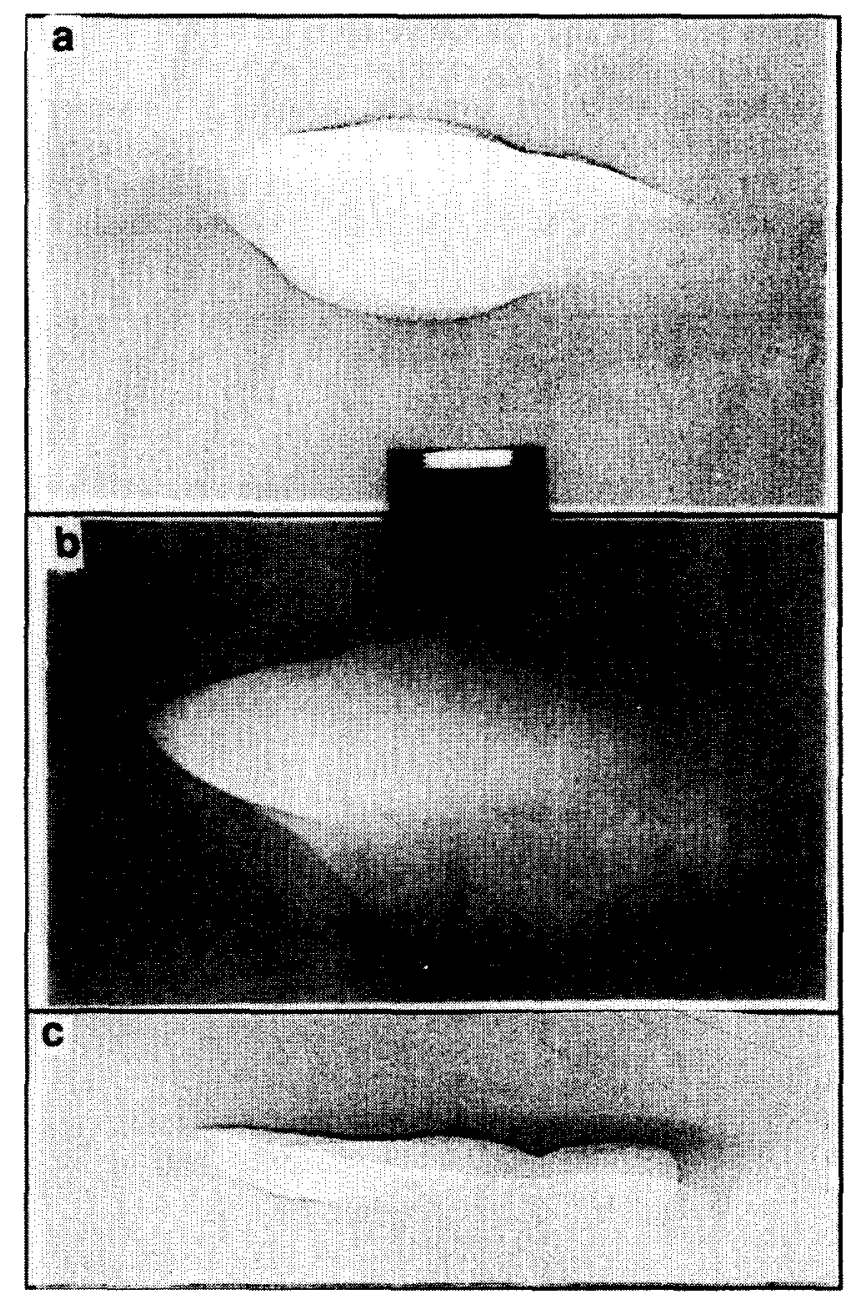

Figure 4 Protocraze development I: (a) sample 1K, cut C1; (b) sample $5 \mathrm{~K}$, cut $\mathrm{C} 2$; (c) sample $3 \mathrm{~K}$, cut $\mathrm{C} 1$. Fatigue stress axis vertical 
consumption near the crack/craze tip during the propagation of large cracks and crazes. This is the most fundamental difference between the initiation and the propagation stages: the structural transformations during the initiation phase occur under relatively low effective stress. Examination at higher magnifications (up to $5 \mathrm{M} \times$ by using the image intensifier) did not detect the presence of fine fibrils (diameter $<10 \mathrm{~nm}$ ) in any of the protocrazes shown in Figure 4. The aspect ratio of the protocrazes in Figures $4 a$ and $b$ is $\sim 3$, however the aspect ratio of the protocrazes in the bulk material will vary depending on the applied stress.

Figure 5 demonstrates that the further development of the initiation process follows the same path as described above; namely growth of a single large void (Figure $5 b$ ), or coalescence of groups of smaller voids along planes perpendicular to the fatigue stress (Figures $5 a$ and $c$ ). It was also found that the general appearance of the protocrazes depends on the cutting mode: under cuts $\mathrm{C} 1$, $\mathrm{C} 2$ and $\mathrm{C} 4$ the void(s) edges are mostly smooth, while under cut $\mathrm{C} 3$ the edges show considerable roughness (Figure $5 b$ ). The rough appearance under cut $\mathrm{C} 3$ is probably an instrumental effect, and as such should not be taken into account when discussing the true shape of the protocrazes.

The protocraze formations similar to those in Figures 2-5 (except Figure 3d) can be found after microtoming appropriate portions of all of the fatigued PC specimens, e.g. $1 \mathrm{~K}, 3 \mathrm{~K}, 5 \mathrm{~K}$ and $7 \mathrm{~K}$. Our observations revealed that the dimensions of the largest void-like formations found in specimens $1 \mathrm{~K}$ and $3 \mathrm{~K}$ are no more than $300-400 \mathrm{~nm}$. The SAXS results reported in reference 27 can be regarded as averaging over the structural formations shown in Figures 2-5 (except Figure 3d). Taking into account the specifics of the SAXS and TEM methods, one can conclude that the results from both methods are

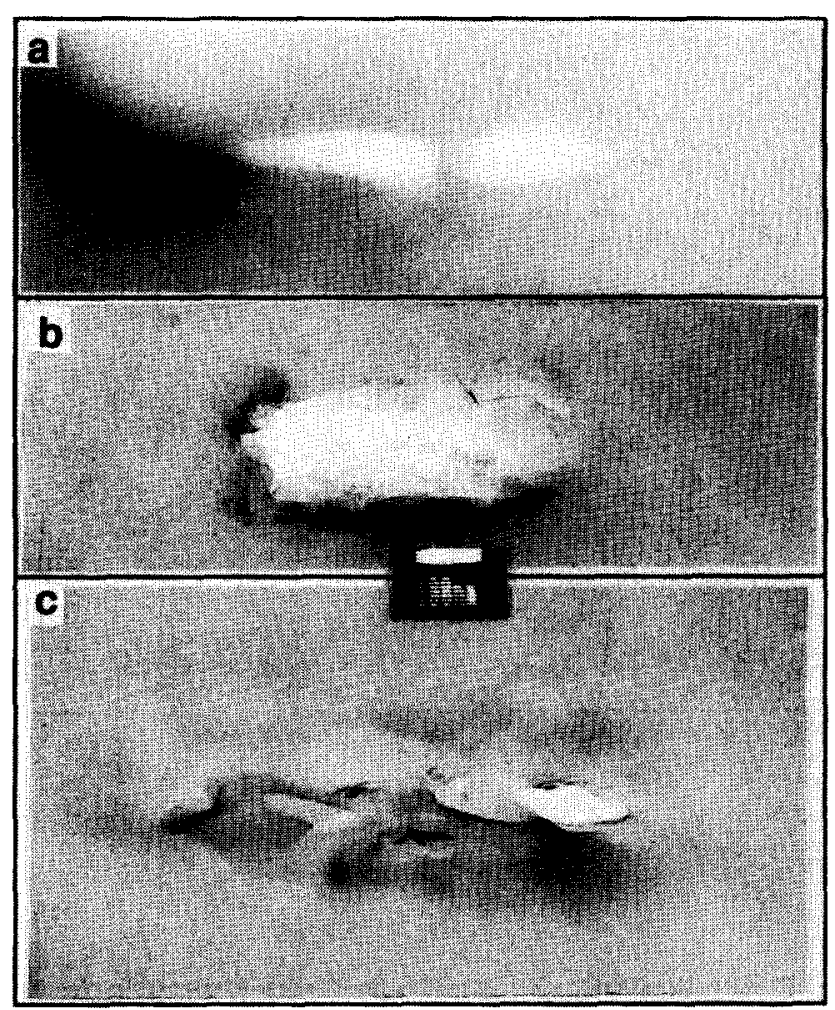

Figure 5 Protocraze development II: (a) sample 3K, cut $\mathrm{Cl}$; (b) sample $5 \mathrm{~K}$, cut $\mathrm{C} 3$; (c) sample $7 \mathrm{~K}$, cut $\mathrm{C} 1$. Fatigue stress axis vertical

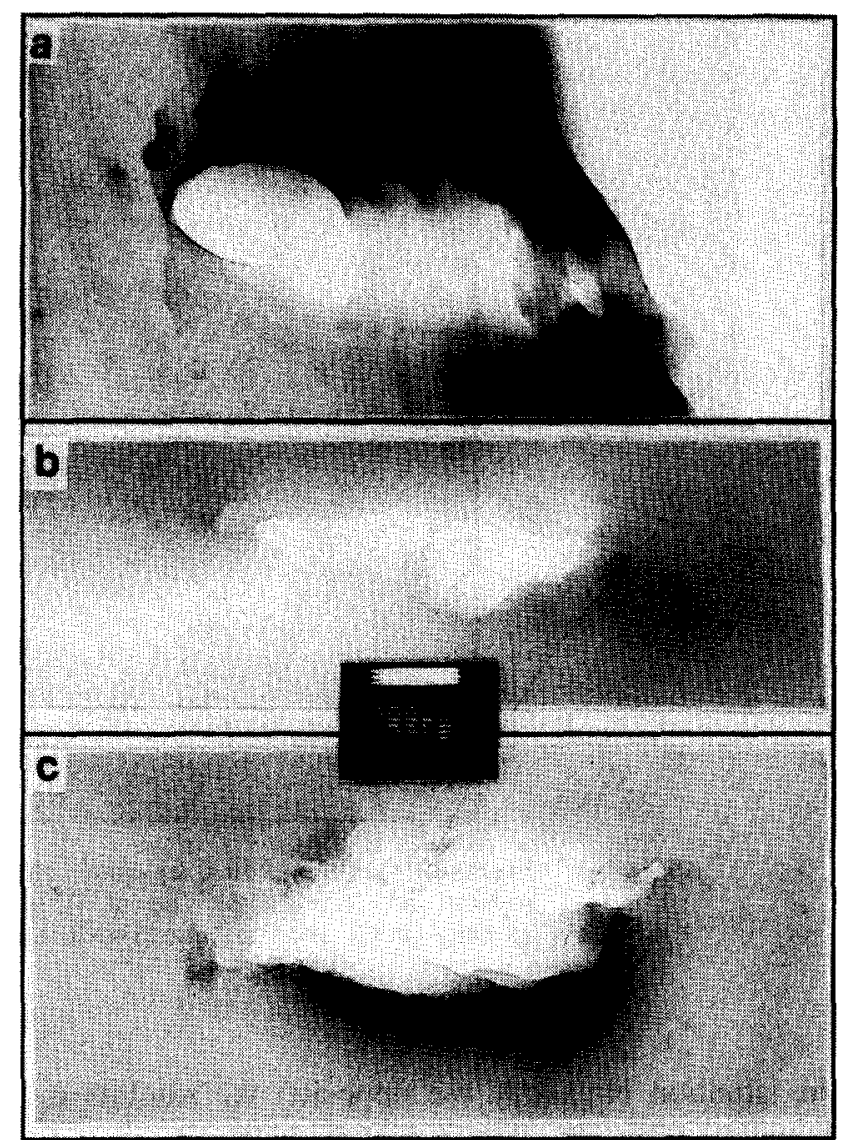

Figure 6 Protocraze to craze transformation: (a) sample $5 \mathrm{~K}$, cut $\mathrm{Cl}$ (b) sample 7K, cut $\mathrm{C} 1$; (c) sample $5 \mathrm{~K}$, cut $\mathrm{C} 3$. Fatigue stress axis vertical

in very good agreement. It is important to point out that the SAXS measurements are performed on bulk samples, which provide additional confirmation that the results from the TEM study are not artefacts introduced by the microtoming and sample handling.

Protocraze to craze transformation (samples $5 K$ and $7 K$ )

The longitudinal dimensions of the protocrazes shown in Figure 6 are in the range $0.5-0.6 \mu \mathrm{m}$. At this size their presence can be detected by optical microscopy, however to determine some of the morphological characteristics by optical microscopy (proto)crazes must be at least in the micrometre range ${ }^{31}$. It should be noted that even if the craze dimensions are several micrometres a detailed picture of the craze structure can be provided only by the electron microscopy methods.

Careful examination of the micrographs reveals that the void-like structure of the protocrazes has a complex topology. The overall shape depends on whether the protocraze is formed near the surface (Figure 6a), or in the bulk of the samples (Figures $6 b$ and $c$ ). The edge of the microtomed slice in Figure $6 a$, which coincides with the side surface of the sample (cut C1), is somewhat curled, but the cavernous character of the protocraze is obvious. The protocraze in Figure $6 b$ is formed by the agglomeration of several voids, and one can speculate that in the place of the largest one was the initiation void, which started growing and induced formation of new voids in a plane perpendicular to the fatigue stress when the number of cycles increases. The rough appearance of the protocraze in Figure $6 c$ is typical for cut $\mathrm{C} 3$. 
Fatigue craze initiation in polycarbonate: H. A. Hristov et al.

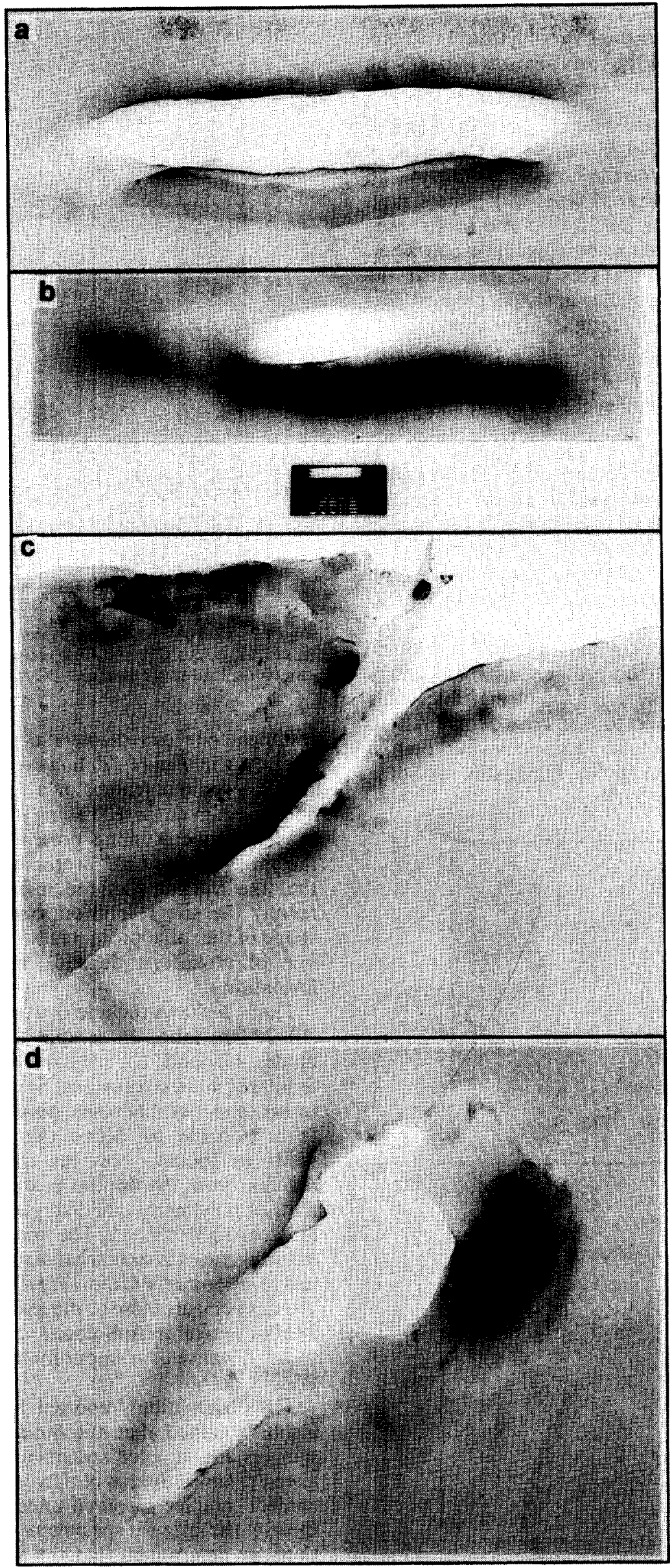

Figure 7 Small crazes/large protocrazes: (a) sample $5 \mathrm{~K}$, cut $\mathrm{C} 1$; (b) sample $7 \mathrm{~K}$, cut $\mathrm{C1}$; (c) sample $5 \mathrm{~K}$, cut $\mathrm{C} 3$; (d) sample $7 \mathrm{~K}$, cut $\mathrm{C} 3$. Fatigue stress axial vertical for (a) and (b), and tilted at an angle of $\sim 45^{\circ}$ for (c) and (d) (note the knife marks in c) 
Figures 7 and 8 show collections of small crazes/large protocrazes with long dimensions varying from 1 to $3 \mu \mathrm{m}$. The overall appearance of the (proto)crazes depends on several factors:

- place of formation - in the bulk (Figures $7 a, b$ and $d$ ) or on the surfaces (Figures $7 c, 8 a$ and $b$ ). The shape of the crazes nucleated inside the samples is mostly of an elongated rotational ellipsoid type, with aspect ratio of $\sim 5$ at this size range (Figures $7 a$ and $b$ ). In some instances the craze shape can be irregular in character, e.g. Figure $7 d$. The short crazes nucleated near the surface are generally triangular in shape, growing in a plane perpendicular to the fatigue stress axis.

- thickness of the microtomed sections - the sections shown in Figures $7 a$ and $8 b$ are $\sim 100 \mathrm{~nm}$ thick, while their counterparts in Figures $7 b$ and $8 a$ are $\sim 300 \mathrm{~nm}$ thick, respectively.

- mode of the microtoming - Figures $7 a$ and $b, 8 a$ and $b$ are under cut $\mathrm{C} 1$, Figure $7 d$ under cut $\mathrm{C} 2$ and Figure $7 c$ under cut $\mathrm{C} 3$. The protocraze in Figure $7 c$ appears to propagate at an angle of $\sim 45^{\circ}$ towards the sample surface but this is a result of the $\mathrm{C} 3$ cutting mode. The knife marks in the lower left corner of the micrograph clearly show that the craze plane is perpendicular to the stress direction.

Despite the different shapes and internal structures, the void-like character of the protocrazes/small crazes is clearly recognizable. The presence of multiple voids gives a spongy appearance to the crazed material in Figure $8 a$. This feature is also vaguely visible on the left side of the broken craze in Figure $8 b$. In some

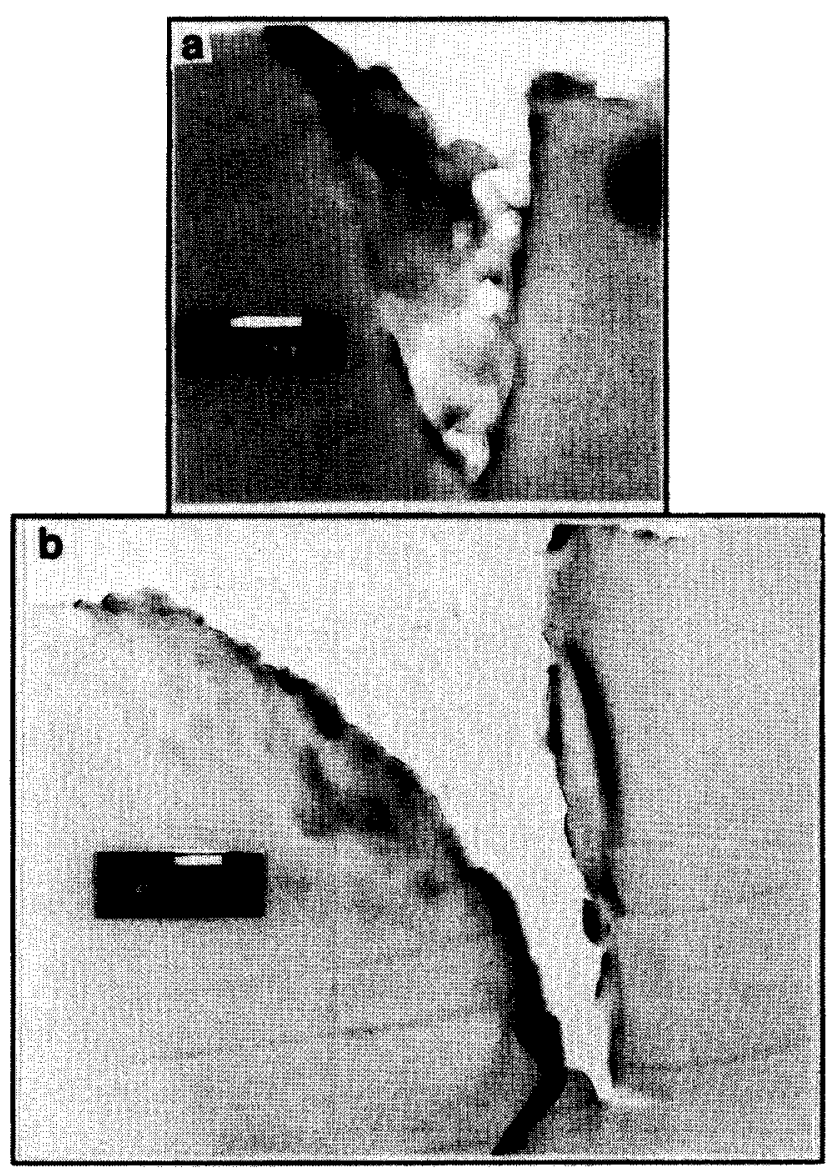

Figure 8 Small surface crazes: (a) sample 5K; (b) sample 7K. Fatigue stress axis horizontal and cut $\mathrm{C} 1$ for both micrographs

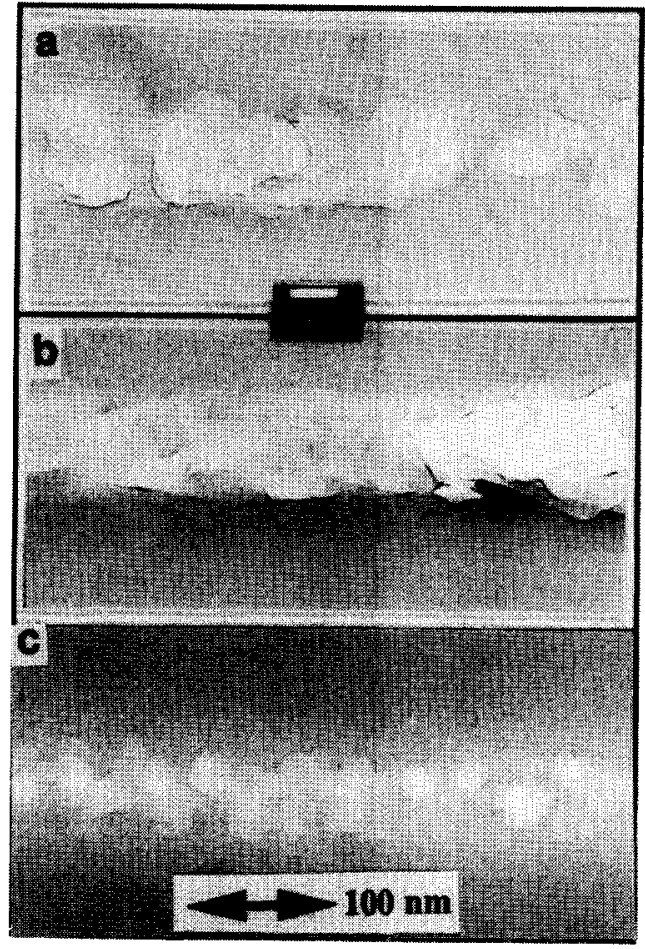

Figure 9 Comparison between large $(10-20 \mu \mathrm{m})$ crazes in sample $7 \mathrm{~K}$ (a, cut $\mathrm{C} 2$; b, cut $\mathrm{C} 1$ ), and high temperature craze, produced under monotonic load in solution cast PC film (c). Stress axis vertical

instances one can detect the presence of small fibrils, as shown in Figure $7 d$, however it is not clear whether this is a genuine feature or a result of the microtoming and/or beam damage. Taking into account that different modes of microtoming present different projections of the three-dimensional protocraze/craze structures, and that the thickness of the microtomed sections affects mainly the size resolution, one can conclude that the shape of the large protocrazes/small crazes in specimens free of inclusions depends mostly on the place of formation.

Figure 9 shows the microstructure of the largest crazes $(10-20 \mu \mathrm{m})$ found in sample $7 \mathrm{~K}$, when microtomed in modes $\mathrm{C} 1$ and $\mathrm{C} 2$ (Figures $9 b$ and $a$, respectively). Similarly to the previous figures one can detect the presence of multiple voids, which may be interconnected. It is possible to define the ligaments between the voids as 'fibrils', however their appearance is very different from the fibrillar morphology observed in thin films of poly(methyl methacrylate), PS and poly(styreneacrylonitrile $)^{6,10,11,14}$. The morphology of the fatigue induced crazes is somewhat similar to the crazes induced in a solution cast PC film (Figure 9c), uniaxially deformed at a temperature close to the glass transition temperature. Similar craze morphologies have been observed, in some instances, in monotonically deformed PS and PVC $^{15,16,32}$.

The observations reported above were compared to results obtained after microtoming non-fatigued control samples and fatigued specimens stained with $\mathrm{OsO}_{4}$. We have examined dozens of thin sections from non-fatigued control samples prepared under identical conditions to those of the fatigued specimens. After extensive investigations we determined that the structural features shown in Figures 2-9 do not result from postfatigue specimen handling or thermal treatment during the sample preparation stage. 


\section{FINAL REMARKS}

As noted in the Introduction, TEM investigations of fatigue craze initiation have not been reported to date. TEM studies of PC films uniaxially deformed at room temperature ${ }^{12,13}$ show that the material yields first and the voiding starts much later in the most severely thinned zones. Thus there is no parallel between the deformation modes during cyclic fatigue of bulk PC and uniaxial deformation of thin films at room temperature. It has been reported that small voids can be detected in $\mathrm{PS}^{15-17}$, prior to craze development at room temperature. The size of the initial voids $(30-60 \mathrm{~nm})$ is in the same range as those shown in Figure 2, but in our specimens we were not able to identify 'nuclei' resulting from localized deformation, or precraze 'furrows'. Crazes in PC are easily produced, under monotonic deformation, in the presence of crazing agents ${ }^{12,13}$ or at temperatures close to the glass transition ${ }^{2,33}$ (Figure 9c). In both cases the environment increases the chain mobility, thus enhances the process of chain disentanglement ${ }^{33}$. The similarity between the fatigue induced crazes and the high temperature crazes suggests that disentanglement of polymer chains should play an important role in the fatigue craze initiation in bulk, amorphous PC. The precise molecular process still awaits more detailed investigation.

The fatigue craze initiation depicted in Figures 2-5 appears to be consistent with at least a part of the model developed by Argon et al. ${ }^{32,34,35}$, describing craze initiation under constant load. The model postulates that the combined effect of the local fluctuations of the stress field and the temperature, with the inherent molecular heterogeneities, leads to development of metastable voids or 'pores' in some small volumes. The number of pores increases with the total time under stress, and after reaching some critical concentration they form a stable craze nucleus (the presence of large scale heterogeneities such as surface scratches or second phase particles accelerates the process). If we assume that the model is applicable (on a qualitative level) in the case of fatigue craze initiation also, then the multiple voids in Figure 3 should represent the first stage when the material develops critical 'porosity'. Logically the voids in Figures 2 and 4 should be regarded as stage II of the process, when stable craze nuclei are created by the coalescence and expansion of the existing pores. We would like to point out that while developing an adequate model of fatigue craze initiation is still an unresolved issue, the above discussed model may serve as a good starting point.

\section{CONCLUSIONS}

In the course of the present investigation we found that TEM and SAXS results obtained from the same set of fatigued PC samples are in very good agreement. The TEM data confirm that fatigue craze initiation starts with the formation of one or more voids, and we can state with certainty that void generation is the main characteristic of the process in bulk, amorphous PC. The growth of the 'protocrazes' into mature crazes is achieved through both the expansion of the existing voids and the generation of new ones in a plane perpendicular to the fatigue stress. The shape and the general appearance of the protocrazes depends mainly on the place of formation, e.g. on the surface or in the bulk of the specimen. We were not able to identify precraze formations (nuclei or furrows), characteristic of craze initiation of PS under constant load and at room temperatures. We observed similarities with crazing of PC films deformed monotonically at temperatures close to the glass transition, which indicates that the fatigue craze initiation process in bulk, amorphous PC may be dominated by the disentanglement of polymer chains.

\section{ACKNOWLEDGEMENTS}

This work is supported by the Department of Energy under grant no. DE-FG02-88ER45366. We are indebted to Professor David C. Martin from the University of Michigan for his helpful comments and discussion. We thank $\mathrm{Mr} \mathrm{C}$. Soles for providing the material for Figure $9 c$, and $\mathrm{Mr} \mathrm{K}$. Mimura for supplying the PC-PS specimens.

\section{REFERENCES}

1 van den Boogaart, A. in 'Physical Basis of Yield and Fracture', Oxford Press, London, 1966, p. 88

Kambour, R. P. and Holik, A.S. J. Polym. Sci. A2 1969, 7, 1393

Kambour, R. P. and Holik, A. S. J. Polym. Sci. A2 1969, 7,
Murray, J. and Hull, D. J. Polym. Sci. A2 1970, 8, 1521

Kambour, R. P. and Russell, R. R. Polymer 1971, 12, 237

Kambour, R. P. Macromol. Rev. 1973, 7, 1

Kramer, E. J. Adv. Polym. Sci. 1983, 52/53,

Friedrich, K. Adv. Polym. Sci. 1983, 52/53, 225

8 Argon, A. S., Cohen, R. E., Gebizioglu, O. S. and Schwier, C. E. Adv. Polym. Sci. 1983, 52/53, 275

9 Kausch, H.-H. in 'Polymer Fracture', Springer, Berlin, 1987, Ch. 9 , p. 332

10 Kramer, E. J. and Berger, L. L. Adv. Polym. Sci. 1990,91/92, 1

11 Argon, A.S. and Cohen, R. E. Adv. Polym. Sci. 1990,91/92, 301

12 Wyzgoski, M. G. and Yeh, G. S. Y. Int. J. Polym. Mater. 1974 3, 149

13 Morgan, R. J. and O'Neal, J. E. Polymer 1979, 20, 375

14 Donald, A. M. and Kramer, E. J. J. Polym. Sci., Polym. Phys. Edn 1982, 20, 899

15 Michler, G. H. Colloid. Polym. Sci. 1985, 263, 462; 1986, 264, 522

16 Michler, G. H. in 'Kunststoff-Mikromechanik', Carl Hanser, Munchen, 1992, Ch. 6, p. 88 (in German)

17 Wellinghoff, S. and Baer, E. J.Macromol. Sci.-Phys. 1975, B11(3), 367

18 Doll, W. Adv. Polym. Sci. 1983, 52/53, 105

19 Doll, W. and Konczoll, L. Adv. Polym. Sci. 1990, 91/92, 137

20 Schirrer, R. Adv. Polym. Sci. 1990, 91/92, 215

21 Takemori, M. T. Adv. Polym. Sci. 1990, 91/92, 263

22 Doll, W. in 'Fractography and Failure Mechanisms of Polymers and Composites' (Ed. A. C. Roulin-Moloney), Elsevier, London, 1989 , Ch. 10 , p. 387

23 Wendorff, J. H. Progr. Coll. Polym. Sci. 1979, 66, 135

24 Brown, H. R., Kramer, E. J. and Bubeck, R. A. J. Polym. Sci., Polym. Phys. Edn 1987, 25, 1765

25 Bubeck, R. A., Buckley, D. J., Kramer, E. J. and Brown, H. R. J. Mater. Sci. 1991, 26, 6249

26 Brown, H. R. Mater. Sci. Rep. 1987, 2, 317

27 Hristov, H. A., Yee, A. F., Xie, L. and Gidley, D. W. Polymer in press

28 Donald, A. M. and Kramer, E. J. J. Mater. Sci. 1981, 16, 2967

29 Liu, L. B., Gidley, D. W. and Yee, A. F. J. Polym. Sci., Polym. Phys. Edn 1992, 30, 231

30 Liu, L. B., Yee, A. F. and Gidley, D. W. J. Polym. Sci., Polym. Phys. Edn 1992, 30, 221

31 Smith, J. W. in 'Fractography and Failure Mechanisms of Polymers and Composites'(Ed. A. C. Roulin-Moloney), Elsevier, London, 1989, Ch. 1, p. 3

32 Argon, A. S. and Hannoosh, J. G. Phil. Mag. 1977, 36(5), 1195

33 Plummer, C. and Donald, A. J. Polym. Sci., Polym. Phys. Edn $1989,27,325$

34 Argon, A. S. J. Macromol. Sci.-Phys. 1973, B8(3-4), 573

35 Argon, A. S. Pure Appl. Chem. 1975, 43, 247 